\title{
Retraction Note: Application of a surgical guide in the extraction of impacted mesiodentes: a randomized controlled trial
}

\author{
Xianghuai Zheng ${ }^{1} \cdot$ Jianjiang Zhao ${ }^{1} \cdot$ Shuguang Liu $^{1} \cdot$ Zhiping Wang $^{1} \cdot$ Bo Jia $^{1} \cdot \mathrm{Xi} \mathrm{Lin}^{2} \mathbb{C}$
}

Published online: 3 March 2022

๑) Springer-Verlag GmbH Germany, part of Springer Nature 2022

\section{Retraction Note: Clinical Oral Investigations \\ https://doi.org/10.1007/s00784-020-03620-x}

The Editor in Chief has retracted this article because it contains material that substantially overlaps with the following article [1]. Xianghuai Zheng has agreed to this retraction but not to the wording of this retraction notice. Jianjiang Zhao, Shuguang Liu, Zhiping Wang, Bo Jia and Xi Lin have not responded to any correspondence from the editor/publisher about this retraction.

The original article can be found online at https://doi.org/10.1007/ s00784-020-03620-x

$\mathrm{Xi}$ Lin

654365980@qq.com

Xianghuai Zheng

xianghuaizheng@yahoo.com

Jianjiang Zhao

zjj2521@sina.com

Shuguang Liu

Dr.liusg@163.com

Zhiping Wang

Sum20004218@139.com

Bo Jia

247459565@qq.com

1 Department of Oral and Maxillofacial Surgery,

Stomatological Hospital, Southern Medical University

(Guangdong Provincial Stomatological Hospital), No. 366,

South of Jiangnan Road, Guangzhou 510280, Guangdong,

People's Republic of China

2 Center of Oral Implantology, Stomatological Hospital,

Southern Medical University (Guangdong Provincial

Stomatological Hospital), No. 366, South of Jiangnan Road,

Guangzhou 510280, Guangdong, People's Republic of China
Reference

[1] Nan Fang Yi Ke Da Xue Xue Baov / Journal of Southern Medical University: Zheng Xianghuai, Lin Xi, Chai Jinyou, Qi Rui, Liu Shuguang. Application of digital positioning guide plate in extraction of impacted supernumerary teeth.J South Med Univ, 2020, 40(6): 907-910. https://doi.org/10.12122/j. issn.1673-4254.2020.06.20

Publisher's note Springer Nature remains neutral with regard to jurisdictional claims in published maps and institutional affiliations. 